QUANTITATIVE INDEXES TO EVALUATE OPERATOR'S BEARING, DIFFICULTY IN TASK, AND MACHINE OPERABILITY WHEN REMOTELY CONTROLLING BACKHOE

\author{
*Shigeomi Nishigaki \\ Mazaran, Co., Ltd., and Kick, Co., Ltd. \\ 3-4-4-208 Kita-Shinjyuku, Shinjyuku-ku \\ Tokyo, Japan 169-0074 \\ (*Corresponding author:sleepingbear@c2mp.com) \\ Katsutoshi Saibara \\ Kick, Co., Ltd. \\ 2-18-11-206 Takasu, Kochi \\ Kochi, Japan 169-0074 \\ Shigeo Kitahara \\ Kumagaigumi, Co., Ltd. \\ 2-1 Tsukudo-cho, Shinjyuku-ku \\ Tokyo, Japan 162-8557 \\ Kenichi Fujino \\ Public Works Research Institute \\ 1-6 Minamihara, Tsukuba \\ Ibaraki, Japan 158-8530
}




\title{
QUANTITATIVE INDEXES TO EVALUATE OPERATOR'S BEARING, DIFFICULTY IN TASK, AND MACHINE OPERABILIY WHEN REMOTELY CONTROLLING BACKHOE
}

\begin{abstract}
This paper presents quantitative indexes to evaluate operator's bearing, difficulty in a task, and machine operability when an operator remotely controls a backhoe, and reports the use cases. The quantitative indexes would give her/him the opportunity to reflect on her/his works, and enable us to gain in-depth insight into the teleoperation, and furthermore give us with promising hints to improve it.
\end{abstract}

\section{KEYWORDS}

Quantitative indexes, Operator's bearing, Difficulty in task, Machine operability, Handling skills of joysticks, Teleoperation of backhoe

\section{INTRODUCTION}

In order to examine teleoperation of a backhoe, there are three key points such as machine operability, difficulty in a task at hand, and operator's bearing in her/his behavioural framework. The bearing means operator's motor skills and attitude when remotely controlling a task at hand (Saibara \& Nishigaki 2011). There is very few information on how to quantitatively evaluate the three key points. Focusing on operator as a controller to operate a backhoe remotely, this study aims to develop quantitative indexes to evaluate the three key points. This paper presents quantitative indexes to evaluate the three key points, and reports the use cases in field experiments executed by Public Works Research Institute (hereafter called PWRI) and in one of the Unzen post-disaster reconstruction projects

\section{QUANTATIVE INDEXES}

\section{Work hypotheses}

In this study, it is supposed that an operator would have excellent resources and capabilities for her/his teleoperation, if the following characteristics might be satisfied.

(1) Movements of backhoe: energy-efficient movements, few changes in acceleration and deceleration, low magnitude of impacts, no tendency to quickly start and stop, and good orientation control; and

(2) Operator's skills: strong learning capability, strong response capability, and good handler of joysticks.

\section{Definition of quantitative indexes}

\section{Level of energy efficiency}

Energy efficiency is defined as an average of each maximum of velocity responses derived from three-axis acceleration responses. The index of energy efficiency is gained by Equation 1.

$$
E F=\frac{1}{3} \sum_{d=1}^{3} \max V_{d}
$$


where $E F$ : index of energy efficiency, $d=1,2,3$ : axis number, and $V_{d}$ : set of $d$ axis velocity.

\section{$\underline{\text { Changeability in acceleration and deceleration }}$}

by Equation 2.

Changeability in acceleration and deceleration is represented by mean cross rate, which is gained

$$
m c r=\frac{1}{3(n-1)} \sum_{d=1}^{3} \sum_{i=1}^{n-1} \frac{\left|\operatorname{sgn}\left(x_{i}-\bar{x}\right)-\operatorname{sgn}\left(x_{i-1}-\bar{x}\right)\right|}{2}
$$

where $m c r$ : mean cross rate, $d=1,2,3$ : axis number, $n$ : number of samples, $x_{i}$ : ith sample, $\bar{x}:$ sample mean, and sgn : sign function.

Magnitude of impact loads

Magnitude of impacts is defined as an average of squared jerk that is gained by Equation 3.

$$
\text { AveJ }^{2}=\frac{1}{3 n} \sum_{d=1}^{3} \sum_{t=1}^{n} J_{d t}{ }^{2},
$$

where $A v e J^{2}$ : average of squared jerk, $d=1,2,3$ : axis number, $n$ : number of samples, $t=1,2, \cdots n$ :

sampling time, and $J_{d t}$ : jerk of $d$ axis at t.

Tendency to quickly start and suddenly stop

Skew and kurtosis of acceleration distribution show some tendency to quickly start and suddenly stop. A negative skew indicates that the tail on the left side of the acceleration distribution is longer than the right side and the bulk of the values lie to the right of the mean. Low kurtosis shows the acceleration distribution has a flat top near the mean rather than a sharp peak, and has thick tails. Therefore, the negative skew and the low kurtosis show that the operator tends to stop the operations suddenly. On the contrary, the positive skew and the low kurtosis show the operator tends to start the operations quickly. If the skew is zero value and the kurtosis is high, it is assumed that the operations might be stable.

\section{Operability of orientation control}

Interquartile (IQR) of curvatures and torsions of three-dimensional acceleration vector movements shows operator's skill at orientation control and machine operability. Large value of the IQR means the bad operability of machine and that the operator is not good at the orientation control. Conversely, the small value shows the ease of operation and the good skill at the orientation control.

\section{Learning capability}

It is well known that, as the number of repetitions of operations for a task at hand increases, the working time to complete the task decreases by a constant percentage. Here, the learning capability is shown by the regression coefficient $\alpha_{1}$ of Equation 4 .

$$
W T=\alpha_{0}+\alpha_{1} \text { Trial }
$$


where WT : working time elapsed, and Trial : number of trials. The estimated regression coefficient $\hat{\alpha}_{1}$ shows the learning capability. As necessary, both of the WT and the Trial are logarithmically transformed.

\section{Difficulty in a task at a hand}

As the target object becomes small, and as the distance covered to the target object becomes long, the task to handle the target becomes difficult (Zhai et al, 2004). In this study, index of the difficulty is represented based on the size of the target, number of trials, and distance covered to the target as Equation 5 .

$$
I D=\frac{D}{W \times L \times H \times \text { trial }},
$$

where $I D$ : index of difficulty; $D:$ distance covered; $W, L$, and $H$ are width, length, and height of the target object, respectively.

$\underline{\text { Response capability }}$ Equation 6.

The relation between the working time elapsed and the index of difficulty is represented by

$$
W T=\beta_{0}+\beta_{1} I D
$$

The estimated regression coefficient $\hat{\beta}_{1}$ shows the response capability. As necessary, both of the $W T$ and the $I D$ are logarithmically transformed.

\section{$\underline{\text { Handling skills of joystick controller }}$}

Movements of the joystick handles pertaining to stick, boom, swing, and bucket might be corelated to one another, since a skilled operator could efficiently and concurrently operate the each joystick handle. Therefore, cross correlation functions among three-axis acceleration responses, which are generated by operating the joystick handles, would be quantitative indexes as to handling skill at joysticks.

\section{USE CASES}

Are reported two use cases of the above quantitative indexes in field experiments executed by PWRI and in one of the Unzen post-disaster reconstruction projects.

\section{Use case 1}

\section{Learning data}

Figure 1 shows two experiments of operating a backhoe remotely were executed in the experiment field of the PWRI. The first experiment was to drive and get over an obstacle of crusher-run stone. The distance driven was about $36 \mathrm{~m}$. The obstacle is of width $3 \mathrm{~m}$, length $2 \mathrm{~m}$, and height $1 \mathrm{~m}$. The second one was to scoop a stockpile of soil on the ground, and to load it and dump it a certain distance away. The soil volume is $9 \mathrm{~m}^{3}$. Two operators joined in the two experiments. One was a middle-aged man (hereinafter referred to operator A). The other was an aged and experienced man (hereinafter referred to operator B). In the two experiments, a three-axis accelerometer was mounted on the stick of the backhoe in order to obtain the learning data. Then, the sampling frequency was $100 \mathrm{~Hz}$. 


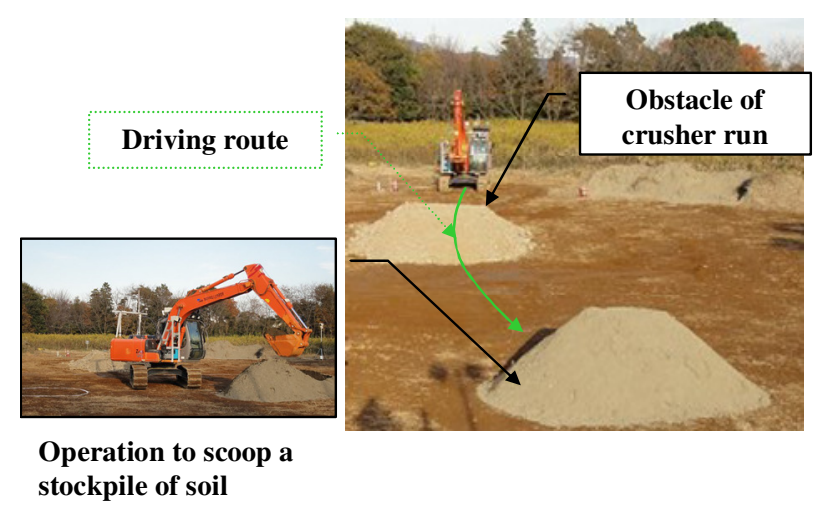

Figure 1 - Two experiments of operating a backhoe remotely

\section{Applications of the quantitative indexes to the two experiments}

Results of the quantitative indexes applied to the first experiments are described below. For want of space of this paper, the results as to the second experiment are left out.

Since there are non-line-of-sight points of climbing route on the obstacle from the monitor in the teleoperation room, it might be difficult for the two operators to steer the backhoe to get over it. So, when travelling uphill at the first time, they had to move the backhoe repeatedly to and fro, and steer it on the left and right. After the several trials, however, they could see the line of sight tracks on the climbing route such as running paths of the crawler belts from the monitor; it became easier for them to get over it. On the other hand, just before and after travelling downhill, were occurred suddenly stopping, jolting, dipping and heaving of the backhoe. Figure 2 shows the obstacle of crusher-run stone after several trials to drive and get over it.

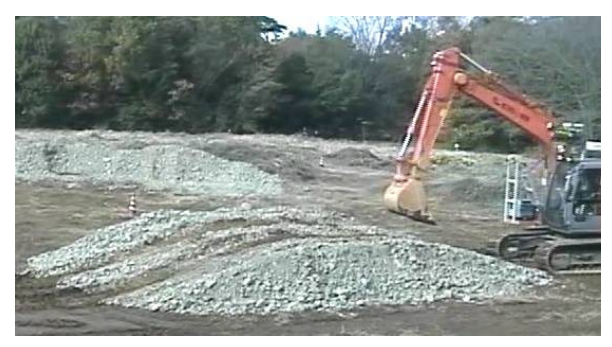

Figure 2 - Obstacle of crusher-run stone after several trials to drive and get over

The operator A has tried his operations nine times. The operator B has tried his operations eight times. There are correlation coefficients of about 0.6 between $\mathrm{Y}$ axis (front-back direction) and $\mathrm{Z}$ axis (vertical direction) responses. As for $\mathrm{X}$ axis (crosswise direction) responses in the final trial, the tails on the right side of these acceleration distributions were longer than the left side, that is, the quick start phenomena. As for $\mathrm{Y}$ axis and $\mathrm{Z}$ axis responses in the final trial, the tails on the left side of these acceleration distributions are longer than the right side, that is, the sudden stop phenomena. It might be considered as the effect of the phenomena of stopping suddenly, jolting, and dipping and heaving. On the other hand, the every distribution has high peak in the final trial, that is, the operation became stable.

Table 1 shows values of the quantitative indexes applied to the first experiment. Table 1 sugests the matters as follows:

- At the initial stage of the experiment, the operator A showed his better skill the operator B, 
- At the final stage, both secures uniform velocity and decrease in degree of chock through the teleoperation,

- Both skills at the orientation control were not improved, and

- Largely speaking, the operator B might have better skills at the teleoperation than the operator A.

Table 1 - Quantitative indexes applied to the first experiment

\begin{tabular}{|c|c|r|r|r|r|r|}
\hline Operator & Times & \multicolumn{1}{|c|}{ Energy } & \multicolumn{1}{c|}{ MCR } & \multicolumn{1}{c|}{ Impact } & Curvature & \multicolumn{1}{c|}{ Torsion } \\
\hline A & 1 & 6.226 & 0.114 & 0.226 & 0.802 & 0.018 \\
\hline A & 9 & 5.097 & 0.125 & 0.147 & 0.81 & 0.158 \\
\hline B & 1 & 6.295 & 0.207 & 0.423 & 1.092 & 0.06 \\
\hline B & 8 & 7.181 & 0.118 & 0.158 & 0.973 & 0.085 \\
\hline
\end{tabular}

Figure 3 shows the regression line of the learning effect to the first experiment. Here, both of the WT and the Trial of Equation 4 are logarithmically transformed. As for the operator A and B, the estimated regression coefficient $\hat{\alpha}_{1} \mathrm{~s}$ are significant and no different between the two at the significance probability 0.05 . It means that both have learning capabilities and there is no difference between the two.

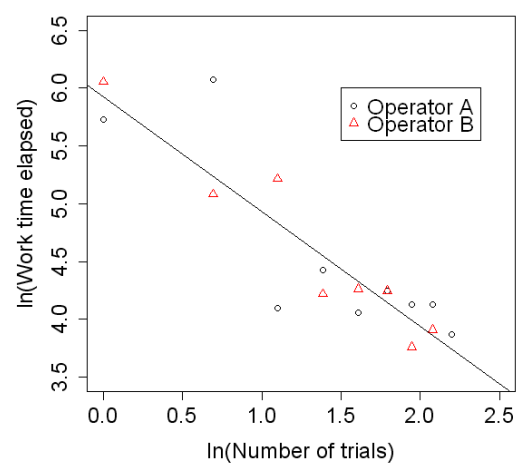

Figure 3 - Regression line of learning effect

Figure 4 also shows the regression line of the response capability to the first experiment. Here, both of the $W T$ and the $I D$ of Equation 6 are logarithmically transformed. As for the operator A and $\mathrm{B}$, the estimated regression coefficient $\hat{\beta}_{1} \mathrm{~s}$ are significant and different between the two at the significance probability 0.05 . The estimated regression coefficient of the operator A is smaller than the operator B's one. It means that although the operator B had longer work time than the operator $\mathrm{A}$ at the initial stage, the operator B has responded to the given task better than the operator A. This result is well consistent with the suggestions inferred from Table 1.

\section{Use case 2}

Learning data

Operating a backhoe, either directly or remotely, is an inherently eye-hand coordination task. The eye-hand coordination means to control eye movements with hand movements, and to handle joysticks along with the use of proprioception of the hands, as processing visual representation of the situations.

As shown in Figure 5, in order to obtain the learning data concerning the handling skills of joystick handles, three-axis accelerometers were mounted on the joysticks to operate a backhoe remotely for loading sediments on a heavy-duty off-road dump truck in one of the Unzen post-disaster 
reconstruction projects. Then the sampling frequency is $20 \mathrm{~Hz}$. The operator here is an experienced and skilled person who is good at compound operations of joystick handles, for examples, boom down as pushing stick out, bucket load as drawing stick in, swing as pushing boom up, and so forth.

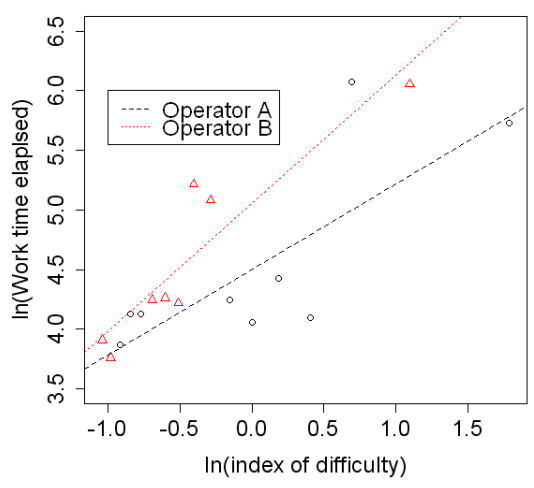

Figure 4 - Regression line of response capability

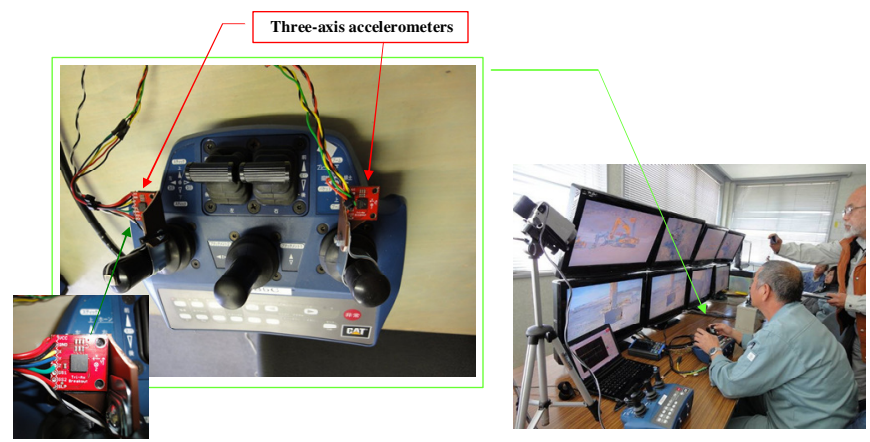

Figure 5 - Joystick with three-axis accelerometers

\section{$\underline{\text { Possibility of cross correlation to represent handling skills at joystick }}$}

Figure 6 shows and the cross correlation functions as to his operations of the joystick handles along a flow of loading operations. It is seen from Figure 6 that there are cross correlations in time lag $1 \sim 40$, this is, $0.05 \sim 2 \mathrm{sec}$ between operations of joystick handles of stick, boom, bucket, and swing.

Especially, from Figure 6, the efficient movements could be conjectured as follows:

(1) From the figure in the top left,

- As drawing stick in for bucket loading efficiently, extracting boom up for efficient swing, and

- As pushing stick out, pushing boom down for quickly positioning bucket's tips on the ground, or efficient bucket dump on the truck bed, and

(2) From the figure in the bottom left,

- As swinging right, that is, when returning to the digging spot, pushing stick out for positioning bucket's tips on the ground quickly, and conversely, and

- As drawing stick in, swinging left for efficiently loading materials on the truck bed, and

(3) From the figures in the top right and the bottom right, are longer time lags between stick out and bucket dump for dumping the material on the truck bed, and between swing right and boom up when returning the digging spot or swing left and boom down for loading on the truck bed. 

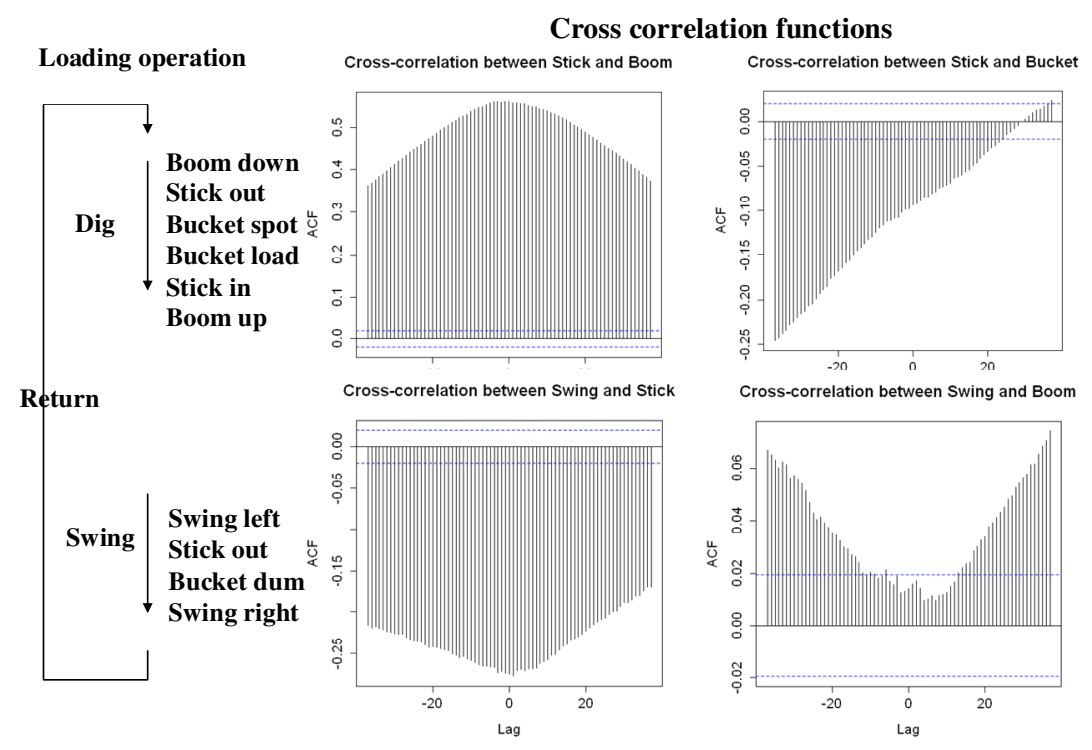

Figure 6 - Flow of loading operations and the cross correlation functions the joystick.

These cross correlation functions mean that the operator might have the efficient handling skills at

\section{REMARKS}

This paper reported the quantitative indexes, which will be able to represent operator's bearing, difficulty in a task and machine operability when operating a backhoe either directly or remotely. If an operator should operate several backhoes or several tasks, then the quantitative indexes could represent the operability of the each backhoe or the difficulty in the each task. Conversely, if several operators should operate a backhoe to handle a task, the quantitative indexes could represent the each operator's motor skill and attitude. The quantitative indexes could be also displayed as numeric or graphic indicators on the monitor of the control station at the noon and the evening.

Taken together, the quantitative indexes will be able to provide not only the operators with the opportunity to reflect on their bearing but also give us in-depth insight into operator's bearing, difficulty of a task, and operability of backhoes, and furthermore give us promising hints to improve backhoe, manmachine interfaces, and the operator's bearing.

As gaining experiences at applications of the quantitative indexes, we will do further research and development on the themes as follows:

- Comparison between skilled and unskilled operators,

- Quantitative index concerning choice-reaction task, and

- Method of operator resources development.

\section{REFERENCES}

Saibara, K., Nishigaki, S. (2011). Driving bearing evaluation system to achieve eco first haulage work, $28^{\text {th }}$ ISARC 2011, pp.520-525.

Zhai, S., Accot, J., Woltjer, R. (2004). Human action laws in electronic virtual worlds - an empirical study of path steering performance in VR, Teleoperation and virtual Environments, Vol. 13, No.2, pp. 113-127. 